\title{
Morphemic ambiguity resolution in Chinese: Activation of the subordinate meaning with a prior dominant-biased context
}

\author{
Yiu-Kei Tsang and Hsuan-ChiH Chen \\ Chinese University of Hong Kong, Shatin, N.T., Hong Kong
}

\begin{abstract}
In the present study, we examined how morphemic ambiguity is resolved using the visual-world paradigm. Participants were presented with Chinese bimorphemic words containing an ambiguous morpheme (analogous to the suffix -ER in TEACHER and TALLER) and performed a visual search task. Their eye-movement patterns during target detection showed that (1) without a prior context, the dominant meaning of an ambiguous morpheme was more available than the subordinate one; (2) with a dominant-biased prior context, the subordinate meaning was still activated; and (3) a subordinate-biased prior context could inhibit the dominant interpretation. Therefore, both the frequency of the intended meaning and the prior contextual biases play a role in morphemic ambiguity resolution. The results are discussed with reference to models of ambiguity resolution and recent proposals of the graded nature of morphological effects.
\end{abstract}

The investigation of lexical ambiguity resolution is one of the major themes in psycholinguistic research. Early studies suggested that initial meaning retrieval is controlled entirely by the properties of an ambiguous word (Hogaboam \& Perfetti, 1975) or entirely by the prior context (Tabossi, 1988), but most contemporary theories assume that both contextual and lexical factors play a role in ambiguity resolution. For instance, according to the reordered-access model (Duffy, Morris, \& Rayner, 1988), all meanings of an ambiguous word are exhaustively activated. Without a biased prior context, the dominant interpretation is more strongly activated than is the subordinate one, but a prior context can facilitate the context-appropriate meaning, although it cannot inhibit the context-inappropriate meaning. When prior context biases toward the dominant interpretation, ambiguity resolution is quick, because effects of meaning frequency and context converge. However, even when the subordinate meaning $(\mathrm{SM})$ is supported by a preceding context $(\mathrm{PC}$, as opposed to succeeding context [SC]), the dominant meaning $(\mathrm{DM})$ is still activated strongly due to its high meaning frequency and the absence of contextual inhibition on the inappropriate meaning. In this condition, extra processing is needed in order to settle the competition between the subordinate-biased context and meaning frequency, creating the subordinate-bias effect (SBE; Pacht \& Rayner, 1993), such that the isolation of the SM of an ambiguous item requires a longer processing time.

The reordered-access model has received extensive support from reading experiments adopting the eyetracking technique (e.g., Sereno, 1995). In particular, as participants read ambiguous words in text, the SBE was observed, despite the presence of a strong subordinate-biased context that should have had a good chance of inhibiting the DM. However, other researchers questioned the effectiveness of the contextual manipulation in these studies and demonstrated an elimination of the SBE by strong contextual constraints (Vu, Kellas, \& Paul, 1998; but see Binder \& Rayner, 1998). To accommodate this finding, Vu et al. proposed the context-sensitive model, according to which prior constraints can inhibit the inappropriate meanings. Thus, there is still some debate regarding whether the PC not only increases the activation level of the context-appropriate meaning but also inhibits the inappropriate ones, such that no competition has to be resolved. This issue is difficult to settle, because the typical eye-tracking procedure in reading experiments does not allow simultaneous monitoring of the activation level of multiple interpretations, which is crucial in validating the exhaustive access assumption in the reordered-access model.

Recently, other researchers have adopted an alternative methodology to study lexical ambiguity resolution. Huettig and Altmann (2007), for example, employed the visual-world paradigm (VWP) and demonstrated meaning coactivation independent of prior context. Chen and Boland (2008) provided further evidence, showing that the DM can be more available than the SM in a neutral context and that a subordinate-biased PC can inhibit the context-inappropriate dominant meaning, even though it is still more available than the baseline. These new findings about ambiguity resolution were made possible by adopting the VWP. 
In a typical VWP experiment, participants' eye movements on a visual display are monitored as the participants hear a speech stimulus. The items on the display share a specific relationship with the research question. For example, a sample display in Huettig and Altmann (2007) contained a pen for writing, a pen for keeping animals, a bicycle, and a bucket as distractors. Previous research showed that participants tended to look at the object they heard, and the fixation proportion (FP) on that object increased when its underlying linguistic representation was more strongly activated (Allopenna, Magnuson, \& Tanenhaus 1998). Therefore, by tracing the FPs on the two "pens," Allopenna et al. concluded that there was a coactivation of meanings because both objects were fixated more than were the distractors. Indeed, Chen and Boland (2008) used differences in FPs across conditions to reveal both the meaning frequency and the contextual effects on ambiguity resolution.

The aforementioned studies mainly concern the fate of the DM in the presence of a subordinate-biased context. The activation of the DM with an unsupportive context was taken as evidence for the exhaustive-access assumption. However, whether the SM of an ambiguous item would be activated with a dominant-biased PC is still unclear. It is possible that the SM would not be activated in this condition, because it is neither frequently used nor supported by the context. Alternatively, according to the reordered-access model, the SM could still be activated, even with an unsupportive context. This is because the model postulates both exhaustive access of multiple meanings and an absence of inhibition on the contextinappropriate meaning. The first goal of the present study was to verify these alternative possibilities with the VWP, which allowed the examination of meaning coactivation in context.

The second goal of the present study was to extend the investigation of ambiguity resolution to the morphemic level. Despite the existence of morphemic ambiguity in languages such as Chinese, Finnish, and English (e.g., $I N$ - in INSANE and INSIDE), how such ambiguity is resolved has rarely been studied. Studies in Dutch suggested that ambiguous morphemes triggered whole-word processing (Bertram, Schreuder, \& Baayen, 2000). In these studies, lexical decisions on words containing homonymic affixes were insensitive to morpheme properties (e.g., base frequency and productivity). However, morphemic decomposition for words containing an ambiguous morpheme was found during natural reading (Kuperman, Bertram, \& Baayen, 2010). Kuperman et al. attributed this difference to the facilitation of morphological parsing when a sentential context was present. As a result, recognition through morphemes became more efficient and outweighed the costs of integrating the ambiguous morphemes. However, it remains unclear how the correct meaning of an ambiguous morpheme is retrieved and integrated with the context.

Chinese is the ideal language to probe morphemic ambiguity resolution, because morphemes are salient orthographic and phonological units in Chinese but not in other languages. This greatly reduces concerns regarding the complexity of segmenting morphologically complex words in alphabetic languages. Our stimuli were Chinese bimorphemic compound words containing an ambiguous morpheme and a contextual morpheme. We manipulated the position of the ambiguous morpheme and the type of contextual bias in order to examine the effects of meaning frequency and context on morphemic ambiguity resolution.

\section{METHOD}

\section{Participants}

Twenty-four undergraduate students at The Chinese University of Hong Kong participated in the experiment voluntarily. All were native Cantonese speakers with normal hearing and vision.

\section{Materials and Design}

Twenty homophonic ambiguous morphemes with two distinctive meanings were first selected. Their relative meaning frequencies were assessed by 20 pilot participants, who wrote down the first associated words that came to mind after they heard the isolated ambiguous morphemes. Those words corresponding to the proposed DM of the ambiguous morpheme $(M=61 \%, S E M=6.18 \%)$ were written down more often than those associated with the SM $(M=11 \%, S E M=2.25 \%) .{ }^{1}$ Each ambiguous morpheme was then combined with 4 contextual morphemes to produce the 80 target bimorphemic nouns under a 2 (context position) $\times 2$ (meaning frequency of the ambiguous morpheme) factorial design. Across conditions, items were matched on various characteristics [context log-frequency: $F(3,76)=1.01$; all other $F \mathrm{~s}<1]$. Sample materials and their corresponding properties are shown in Table 1.

Eighty object pictures, each corresponding to a target bimorphemic noun, were then constructed to form visual displays for the visual search task. Each display contained three objects (a target, a competitor, and a distractor) arranged in a V-shape or an invertedV-shape, with target location rotated across trials. The target and the competitor in each trial were complementary, such that one of them corresponded to the DM and the other corresponded to the SM of the same homophomic morpheme at the same position within a bimorphemic word. For instance, for the SC-SM target “蜂巢” (comb), the competitor would be the SC-DM object “風筝” (kite). The distractor items from other stimulus sets were used to ensure that each object appeared equally often as a target, competitor, and distractor across trials. Eighty filler displays were also constructed in a similar fashion, except that this construction did not contain an object corresponding to the spoken target. The 160 trials were split into four blocks. Each block contained 20 fillers and 5 experimental trials per condition. Objects and target morphemes did not repeat within a block. Eight practice trials were prepared in a similar fashion.

\section{Procedure}

Participants were tested individually. They were seated about $50 \mathrm{~cm}$ from the computer screen. At this distance, each object on the display spanned about $5^{\circ} \times 5^{\circ}$, and the intercenter distance between objects was about $10^{\circ}$. Participants' eye movements were recorded by an Eyelink-1000 desktop system. Viewing was binocular, but recording was made of the right eye only. A chinrest was used to minimize head movements. Calibration ensured the accuracy of fixation recording to be within $0.5^{\circ}$. Participants were then given the practice trials. In each trial, a central fixation point first appeared on the screen. After this point was fixated stably, a spoken target and a visual display were presented simultaneously, and the participants had to decide whether the spoken target was on the display, which remained on the screen until a response was made or after 2,500 msec. The participants' eye movements and keypressing responses were recorded in each trial. After practice, 
Table 1

Sample Materials, Their Properties, and the Mean Reaction Times (in Milliseconds) and Error Rates for the Detection Task

\begin{tabular}{|c|c|c|c|c|c|c|c|c|c|c|c|c|c|c|c|}
\hline \multirow[b]{2}{*}{ Syllable } & \multirow[b]{2}{*}{ Morpheme } & \multirow[b]{2}{*}{ Condition } & \multirow{2}{*}{$\begin{array}{l}\text { Sample } \\
\text { Disyllabic } \\
\text { Word }\end{array}$} & \multicolumn{2}{|c|}{$\begin{array}{l}\text { Context } \\
\text { Log- } \\
\text { Frequency }\end{array}$} & \multicolumn{2}{|c|}{$\begin{array}{l}\text { Whole } \\
\text { Word } \\
\text { Familiarity }\end{array}$} & \multicolumn{2}{|c|}{$\begin{array}{c}\text { Acoustic } \\
\text { Duration } \\
\text { of First } \\
\text { Morpheme }\end{array}$} & \multicolumn{2}{|c|}{$\begin{array}{l}\text { Acoustic } \\
\text { Duration } \\
\text { of Second } \\
\text { Morpheme }\end{array}$} & \multicolumn{2}{|c|}{$\begin{array}{l}\text { Reaction } \\
\text { Time }\end{array}$} & \multicolumn{2}{|c|}{ Error Rate } \\
\hline & & & & $M$ & $S E M$ & $M$ & $\overline{S E M}$ & $M$ & SEM & $M$ & SEM & $M$ & SEM & $M$ & $\overline{S E M}$ \\
\hline \multirow[t]{4}{*}{ /fung1/ } & 風 Wind & SC-DM 風筆 & $\begin{array}{l}\text { /fung1zaang1/ } \\
\text { wind-kite } \\
\text { Kite }\end{array}$ & 2.95 & 0.15 & 3.98 & 0.22 & 564 & 29.85 & 626 & 21.74 & 1,622 & 16.76 & 18.12 & 1.77 \\
\hline & & PC-DM 旋風 & $\begin{array}{l}\text { /syun4fung1/ } \\
\text { whirl-wind } \\
\text { Tornado }\end{array}$ & 3.21 & 0.16 & 4.01 & 0.19 & 588 & 21.57 & 620 & 30.58 & 1,611 & 15.02 & 9.17 & 1.33 \\
\hline & 蜂 Bee & SC-SM 蜂巢 & $\begin{array}{l}\text { |fung1caau4/ } \\
\text { bee-nest }\end{array}$ & 3.25 & 0.22 & 3.77 & 0.21 & 568 & 27.83 & 620 & 23.46 & 1,694 & 19.13 & 19.79 & 1.83 \\
\hline & & PC-SM 蜜蜂 & $\begin{array}{l}\text { /mat6fung1/ } \\
\text { honey-bee } \\
\text { Honeybee }\end{array}$ & 3.30 & 0.14 & 3.86 & 0.13 & 575 & 30.77 & 628 & 27.98 & 1,661 & 15.97 & 16.67 & 1.71 \\
\hline
\end{tabular}

Note-SC-DM, succeeding context-dominant meaning; PC-DM, preceding context-dominant meaning; SC-SM, succeeding context-subordinate meaning; PC-SM, preceding context-subordinate meaning. Whole-word familiarity was rated on a 6-point Likert scale.

the participants were given the four experimental blocks, with a 3-min break between blocks. Block order was counterbalanced across participants. Item order within a block was randomized. The whole experiment lasted about $1 \mathrm{~h}$.

\section{RESULTS}

The following types of responses were excluded from data analyses: Incorrect responses; no response within $2,500 \mathrm{msec}$; reaction times (RTs) $<1,000 \mathrm{msec}$ (i.e., spoken target durations were generally $>1,000 \mathrm{msec}$ ); and trials in which participants responded before having fixated on the target. In sum, $15.88 \%$ of the data were removed. Table 1 also shows the mean RTs and error rates (ERs) of target detection for the remaining trials. In the present study, we evaluated the fixed effects of meaning frequency of an ambiguous morpheme and context position while treating participants and items as crossed random effects in mixed models. Our analyses were carried out with the lmer program (lme 4) in R 2.11.1 (R Development Core Team, 2010). The keypressing RTs and the eye-movement data were analyzed using the linear mixedeffects (lme) model, (Baayen, 2008), whereas the mixed logit model was used to analyze the ERs (Jaeger, 2008). ${ }^{2}$ For the lme models, we report regression coefficients ( $b s$, effects relative to the intercept), standard errors ( $S E \mathrm{~s})$, and $p$ values estimated using posterior distributions for model parameters obtained by Markov chain Monte Carlo sampling. For the mixed logit model, we reported the Wald $z$ values and $p$ values based on Laplace approximation.

\section{Analyses on Keypressing Responses}

For RTs, only the main effect of meaning frequency was significant $(b=36.21, S E=7.76, p=.0001)$. Target detection was slower when the ambiguous morpheme in the target corresponded to the SM rather than to the DM. For ERs, the main effects of meaning frequency $(b=0.21$, $S E=.067, z=3.18, p<.005)$ and context position $(b=$ $0.27, S E=.066, z=4.00, p=.0001)$ were significant.
Moreover, the interaction between meaning frequency and context position was also significant $(b=0.15, S E=$ $.067, z=2.29, p<.05)$. This interaction can be attributed to the lower ER in the PC-DM condition than in the other conditions. No other effects were found.

\section{Eye-Movement Analyses}

The mean FPs on each object during every $100-\mathrm{msec}$ time window for the SC and PC conditions are shown in Figures 1 and 2, respectively. In all conditions, object fixations started at around $200 \mathrm{msec}$, which was expected, given the time needed for saccade planning and execution. Moreover, participants fixated mostly on the target object when they produced overt responses at around $1,600 \mathrm{msec}$. Overall, participants established stable fixations on targets (defined as reaching a $10 \%$ difference in FPs between the target and the competitor for three consecutive time windows) ${ }^{3}$ faster in the PC than in the SC conditions ( $b=47.58, S E=8.31, p=.0001)$. They also looked stably on the DM targets earlier than on the SM targets $(b=22.36, S E=8.30, p<.01)$. Therefore, the DM of the ambiguous morpheme is more available, but a $\mathrm{PC}$ can also facilitate meaning retrieval.

Table 2 shows the mean FPs on competitors and distractors (object identity) at each level of meaning frequency, context position, and time window. These factors and the corresponding interactions were included in the lme model to examine whether the competitors were more highly activated than the baseline distractors (suggesting meaning coactivation) and whether this meaning coactivation was present only in specific conditions. To illustrate the corresponding activations over time for both competitors and distractors, we subtracted distractor fixations from competitor fixations (positive values reflect higher competitor fixations than distractor fixations) and present the results in Figure 3.

There were significant main effects for object identity $(b=2.33, S E=.304, p=.0001)$, meaning frequency $(b=1.12, S E=.305, p=.0001)$, context position $(b=$ 


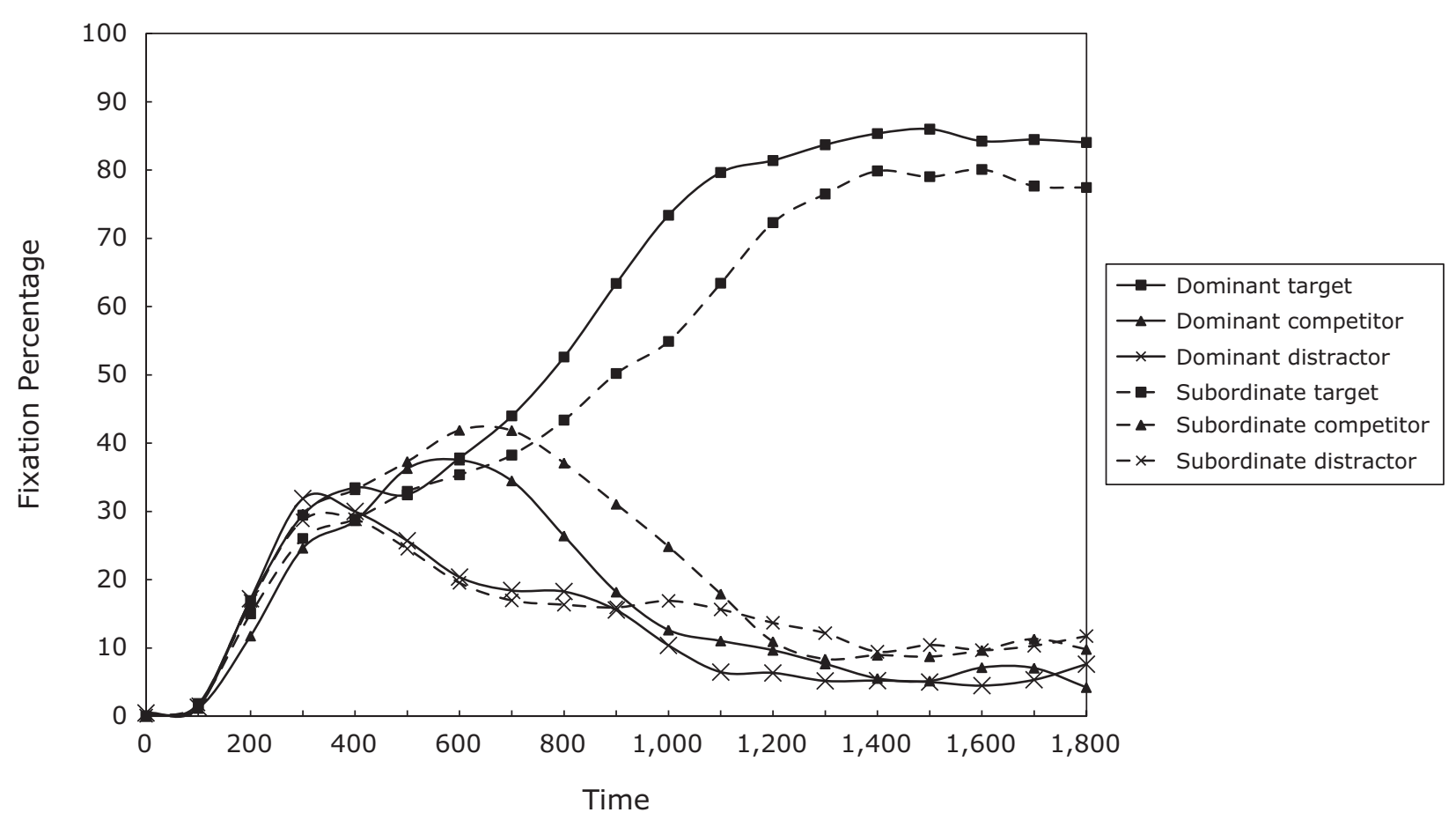

Figure 1. Fixation percentages on different objects over time in the succeeding context conditions.

1.06, $S E=.305, p<.0005)$, and time window $(b=$ $6.47, S E=.303, p=.0001)$. Overall, FPs were higher for competitors, when the ambiguous morpheme in the target corresponded to the SM in the SC conditions and in the first time window. More important, the four-way interaction was significant $(b=0.80, S E=.304, p<$
.01 ), suggesting a complex interplay among the factors in determining meaning activation. To examine the conditions under which meaning coactivation occurred, we conducted lme modeling in the SC and PC conditions separately. Furthermore, to compensate for the inflation of Type I error due to multiple model fitting, we only

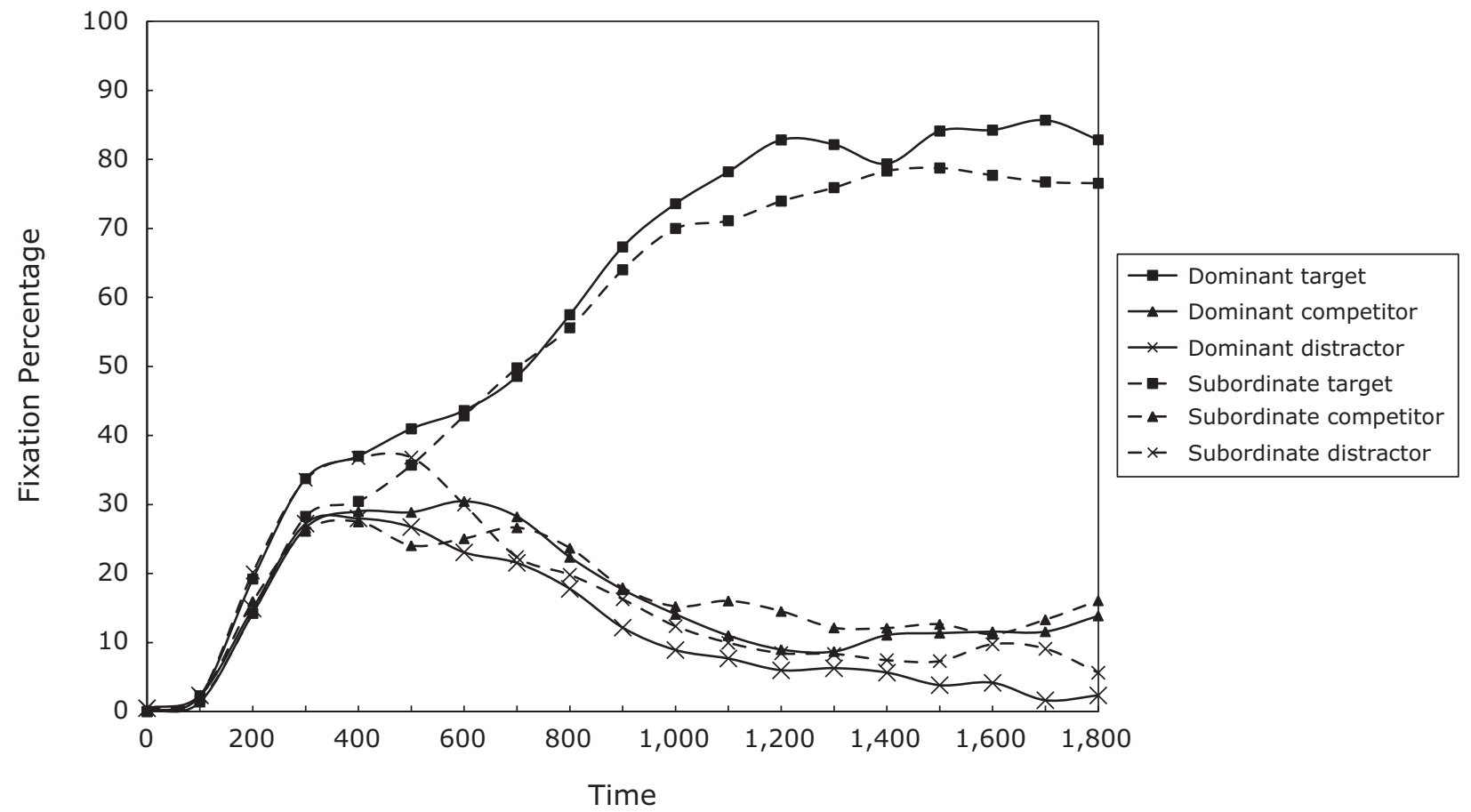

Figure 2. Fixation percentages on different objects over time in the preceding context conditions. 
Table 2

Mean Fixation Proportions on Competitors and Distractors

\begin{tabular}{|c|c|c|c|c|c|c|c|c|c|c|c|c|c|c|c|c|}
\hline & \multicolumn{4}{|c|}{$\begin{array}{c}\text { SC-DM } \\
\text { 風箏 (kite) }\end{array}$} & \multicolumn{4}{|c|}{$\begin{array}{c}\text { SC-SM } \\
\text { 蜂巢 (comb) }\end{array}$} & \multicolumn{4}{|c|}{$\begin{array}{c}\text { PC-DM } \\
\text { 旋風 (tornado) }\end{array}$} & \multicolumn{4}{|c|}{$\begin{array}{c}\text { PC-SM } \\
\text { 蜜蜂 (bee) }\end{array}$} \\
\hline & \multicolumn{2}{|c|}{ Competitor } & \multicolumn{2}{|c|}{ Distractor } & \multicolumn{2}{|c|}{ Competitor } & \multicolumn{2}{|c|}{ Distractor } & \multicolumn{2}{|c|}{ Competitor } & \multicolumn{2}{|c|}{ Distractor } & \multicolumn{2}{|c|}{ Competitor } & \multicolumn{2}{|c|}{ Distractor } \\
\hline & $M$ & $\overline{S E M}$ & $M$ & $\overline{S E M}$ & $M$ & SEM & $M$ & $\overline{S E M}$ & $M$ & SEM & $M$ & SEM & $M$ & $\overline{S E M}$ & $M$ & $S E M$ \\
\hline rst morp & 6.21 & 1.28 & 20.35 & 1.15 & 29.84 & 1.31 & 19.21 & 1.14 & 23.24 & 1.10 & 0.09 & 1.07 & 21.34 & 1.13 & 25.99 & 1.22 \\
\hline Second morpheme & 12.58 & 0.99 & 9.54 & 0.83 & 19.10 & 1.24 & 12.58 & 1.00 & 11.66 & 0.95 & 9.34 & 0.82 & 12.92 & 1.05 & 10.87 & 0.94 \\
\hline
\end{tabular}

Note-Conditions: SC-DM, succeeding context-dominant meaning; SC-SM, succeeding context-subordinate meaning; PC-DM, preceding context-dominant meaning; PC-SM, preceding context-subordinate meaning. First morpheme, 200-799 msec; second morpheme, $800-1,399$ msec.

report effects with $p<.0083$ (in total, there were six submodels).

In the $\mathrm{SC}$ conditions, the competitors were fixated more than were the distractors $(b=3.12, S E=.402, p=$ .0001 ), suggesting meaning coactivation. There were more fixations on both the competitors and distractors when the target corresponded to the $\mathrm{SM}(b=1.42, S E=.408$, $p<.001$ ), reflecting the more difficult target detection in the SC-SM condition. Moreover, there was a strong time window effect $(b=5.40, S E=.403, p=.0001)$. Fixations on the competitors/distractors generally decreased in the second time window, probably because participants had found the target and were fixating on it. Finally, the two-way interaction between meaning frequency and object identity was marginally significant $(b=1.07, S E=$ $.403, p=.0088)$. There was a tendency for a larger difference between competitor fixations and distractor fixations when the target corresponded to the SM $(8.58 \%$ vs. $4.45 \%$ ). The DM competitor tended to be more available than the SM competitor. No other effects were significant at the adjusted $p$ level ( $p$ s $=.017$ to .347 ).
In the PC conditions, the three-way interaction among object identity, meaning frequency, and time window was significant $(b=1.18, S E=.407, p<.005)$. Therefore, we further split the data on the basis of meaning frequency of the ambiguous morpheme. In the PC-DM condition, the competitors were fixated more than were the distractors $(b=2.45, S E=.538, p=.0001)$. There was also a clear decrease in competitor/distractor fixations in the second time window $(b=6.95, S E=.528, p=.0001)$. The interaction was not significant $(p=.89)$. The above-baseline activation of competitors was thus stable across time windows. In the PC-SM condition, however, there was a strong interaction between object identity and time window $(b=2.38, S E=.601, p=.0001)$. There were more distractor fixations than competitor fixations in the first time window $(b=2.53, S E=.883, p<.005)$, whereas the difference was not significant in the second time window $(b=0.83, S E=.69, p=.22)$. In other words, the context-inappropriate DM was initially inhibited in the PC-SM condition. This inhibition was neutralized in the second time window.

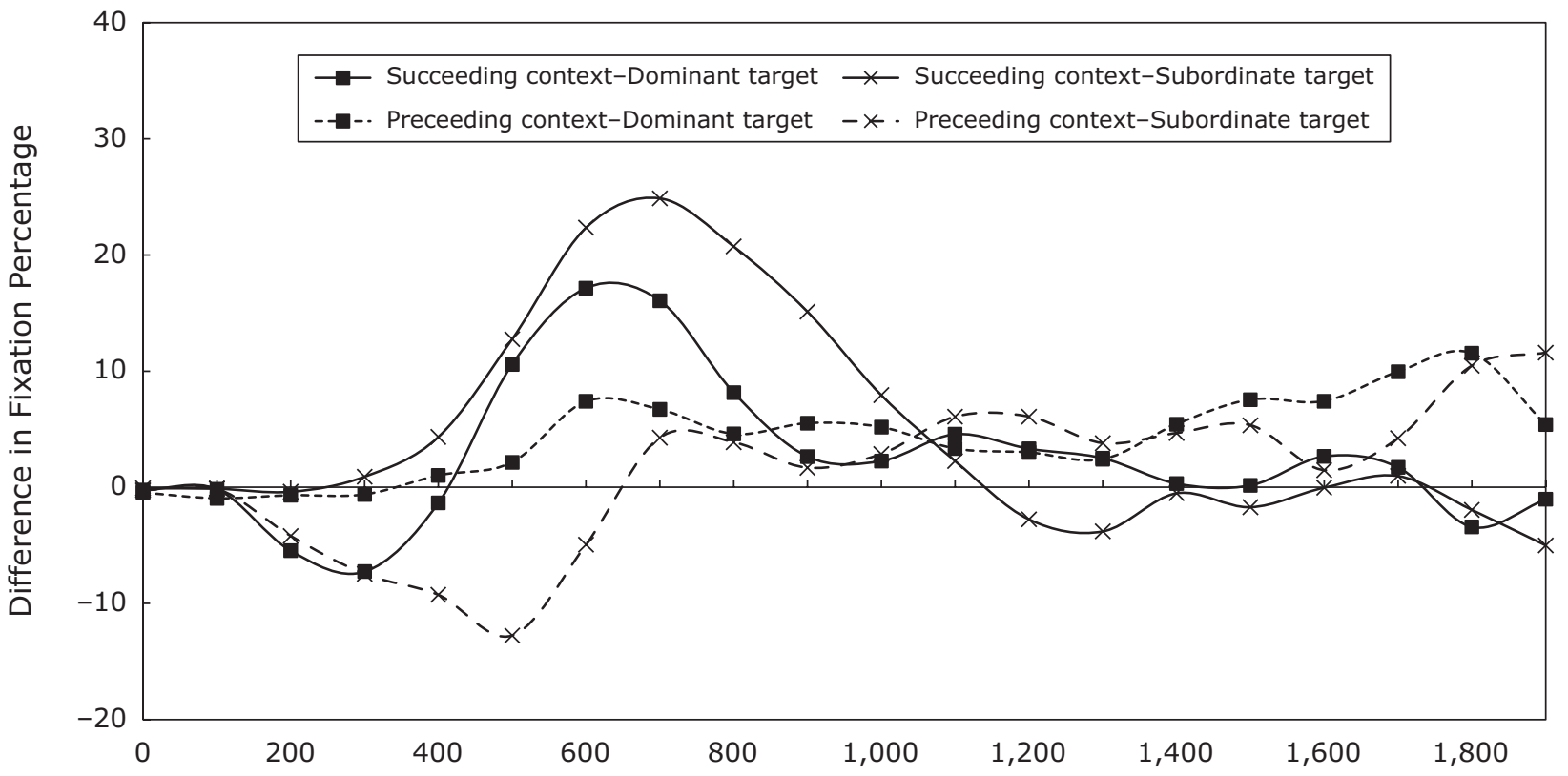

Time

Figure 3. Difference in fixation percentages between competitors and distractors in the four experimental conditions. 


\section{DISCUSSION}

In the present experiment, participants performed a visual search task while their eye movements on various displayed objects were monitored. The target to be detected contained an ambiguous morpheme and a context morpheme. Results suggested that the DM was accessed faster and a PC morpheme could facilitate meaning retrieval, leading to more rapid and accurate target fixations and detection responses. More important, participants' eye-movement patterns also reflected that both meaning frequency and context contribute to ambiguity resolution at the morphemic level. Consistent with findings at the lexical level (Chen \& Boland, 2008; Huettig \& Altmann, 2007), without a prior biasing context, meaning coactivation occurred. But the DM was more available than the SM, as indexed by the significantly lower competitor/distractor fixations in the SC-DM relative to the $\mathrm{SC}-\mathbf{S M}$ condition and the tendency of a larger difference between competitor and distractor fixations in the SC-SM condition.

A unique finding of the present study is the demonstration that the SM is activated in the PC-DM condition. This strongly supports the exhaustive-access assumption, such that, during natural, noninterrupted comprehension, multimeaning activation occurs, even in the least possible condition where meaning frequency and context both favor the dominant interpretation.

Furthermore, meaning coactivation was observed in three out of four conditions. The only exception was in the PC-SM condition, in which the competitors with the DM were completely inhibited by a subordinate-biased $\mathrm{PC}$ in the first time window. Contextual constraint on inappropriate meanings was also observed in previous studies (e.g., Chen \& Boland, 2008). However, the dominant competitors in these studies were just partially inhibited. These competitors were still more available than were the unrelated distractors. Although further studies are needed to investigate why complete inhibition was seen in this (but not in previous) studies, our data clearly suggest that a valid model of ambiguity resolution should incorporate both the assumption of exhaustive access and the inhibition of context-inappropriate meaning by PC.

The present results are also consistent with previous studies showing that ambiguous morphemes could still play an important role in lexical access during comprehension (Kuperman et al., 2010). Moreover, meaning frequency influences the ease of morpheme integration, such that the DM could be more easily recognized. This fits well with the accumulating evidence on the graded nature of morphological effects (Hay \& Baayen, 2005), which suggests that the size of morphological effects in word recognition is dependent on continuous dimensions, such as productivity and semantic transparency.

There are two major theoretical accounts for understanding the graded nature of morphological effects. One is to maintain the localist representations of morphemes and to attribute the graded effect to processing (Taft \& Nguyen-Hoan, 2010). For example, separate representa- tions can exist for each meaning of an ambiguous morpheme, and the activation threshold for the DM is lower than that for the SM. Another account is to assume that the morphological structure is graded (Gonnerman, Seidenberg, \& Andersen, 2007). According to this view, morphological structure emerges because of the convergence between form and meaning. Ambiguous morphemes represent a special case, in which the same form corresponds to two distinct but productive meanings. When the system encounters an ambiguous morpheme, the initial output will be a blend of multiple meanings, with a slight bias toward representing the more frequently used DM. As a result, it will take longer to overcome the initial bias and converge on the SM. Although the present data are unable to verify these two accounts, investigating how ambiguous morphemes are processed may shed light on this issue in the future.

To conclude, the present study demonstrates that meaning frequency and context work jointly in resolving morphemic ambiguity. We also extend previous findings by showing that meaning coactivation occurs regularly, even when the inappropriate meaning is the subordinate one. In addition, context modulates the effects of meaning frequency. Without a biased PC, the DM is more available than is the SM, but an unsupportive PC can suppress the inappropriate DM. Taken together, these results are consistent with the graded nature of morphological effects.

\section{AUTHOR NOTE}

This research was supported by a grant from the Research Grants Council of the Hong Kong Special Administrative Region, China (CUHK4142/04H). We thank Matt Goldrick, Victor Kuperman, and Keith Rayner for constructive comments on an earlier version of the manuscript. Correspondence concerning this article should be sent to Y.-K. Tsang or H.-C. Chen, Department of Psychology, Chinese University of Hong Kong, Shatin, N.T., Hong Kong (e-mail: yktsang@psy .cuhk.edu.hk or hcchen@psy.cuhk.edu.hk).

Note-Accepted by Cathleen M. Moore's editorial team.

\section{REFERENCES}

Allopenna, P. D., Magnuson, J. S., \& Tanenhaus, M. K. (1998). Tracking the time course of spoken word recognition using eye movements: Evidence for continuous mapping models. Journal of Memory \& Language, 38, 419-439.

BAAYEN, R. H. (2008). Analyzing linguistic data: A practical introduction to statistics. Cambridge: Cambridge University Press.

BARR, D. J. (2008). Analyzing "visual world" eyetracking data using multilevel logistic regression. Journal of Memory \& Language, 59, 457-474.

Bertram, R., Schreuder, R., \& Bayyen, R. H. (2000). The balance of storage and computation in morphological processing: The role of word formation type, affixal homonymy, and productivity. Journal of Experimental Psychology: Learning, Memory, \& Cognition, 26, 489-511.

Binder, K. S., \& RAYNer, K. (1998). Contextual strength does not modulate the subordinate bias effect: Evidence from eye fixations and selfpaced reading. Psychonomic Bulletin \& Review, 5, 271-276.

Chen, L., \& Boland, J. E. (2008). Dominance and context effects on activation of alternative homophone meanings. Memory \& Cognition, 36, 1306-1323.

Duffy, S. A., Morris, R. K., \& RAYNer, K. (1988). Lexical ambiguity and fixation times in reading. Journal of Memory \& Language, 27, 429-446. 
Gonnerman, L. M., Seidenberg, M. S., \& Andersen, E. S. (2007). Graded semantic and phonological similarity effects in priming: Evidence for a distributed connectionist approach to morphology. Journal of Experimental Psychology: General, 136, 323-345.

HAY, J. B., \& BAAYEN, R. H. (2005). Shifting paradigms: Gradient structure in morphology. Trends in Cognitive Sciences, 9, 342-348.

Hogaboam, T. W., \& Perfetti, C. A. (1975). Lexical ambiguity and sentence comprehension. Journal of Verbal Learning \& Verbal Behavior, 14, 256-274.

Huettig, F., \& Altmann, G. T. M. (2007). Visual-shape competition during language-mediated attention is based on lexical input and not modulated by contextual appropriateness. Visual Cognition, 15, 9851018.

JAEGER, T. F. (2008). Categorical data analysis: Away from ANOVAs (transformation or not) and towards logit mixed models. Journal of Memory \& Language, 59, 434-446.

Kuperman, V., Bertram, R., \& Baayen, R. H. (2010). Processing trade-offs in the reading of Dutch derived words. Journal of Memory \& Language, 62, 83-97.

Mirman, D., Dixon, J. A., \& Magnuson, J. S. (2008). Statistical and computational models of the visual world paradigm: Growth curves and individual differences. Journal of Memory \& Language, 59, 475-494.

PACHT, J. M., \& RAYNER, K. (1993). The processing of homophonic homographs during reading: Evidence from eye movement studies. Journal of Psycholinguistic Research, 22, 251-271.

R Development Core Team (2010). R: A language and environment for statistical computing. Vienna: R Foundation for Statistical Computing.
Sereno, S. C. (1995). Resolution of lexical ambiguity: Evidence from an eye movement priming paradigm. Journal of Experimental Psychology: Learning, Memory, \& Cognition, 21, 582-595.

TABossi, P. (1988). Accessing lexical ambiguity in different types of sentential contexts. Journal of Memory \& Language, 27, 324-340.

TAFT, M., \& NGUYEN-HoAn, M. (2010). A sticky stick? The locus of morphological representation in the lexicon. Language \& Cognitive Processes, 25, 277-296.

Vu, H., Kellas, G., \& Paul, S. T. (1998). Sources of sentence constraint on lexical ambiguity resolution. Memory \& Cognition, 26, 979-1001.

\section{NOTES}

1. Some participants produced an associated word that was unrelated to either the dominant or the subordinate meaning, so the values do not sum up to $100 \%$.

2. Note that the data obtained in VWP need to be handled with special care due to the time-varying nature and interdependence of fixations on various objects. Such data can be analyzed using other recently developed methods, including growth-curve modeling (Mirman, Dixon, \& Magnuson, 2008) and multilevel logistic regression (Barr, 2008). However, which method is the most appropriate is still under debate.

3. This is admittedly a somewhat arbitrary criterion, but fixations on targets generally showed an increasing trend when this $10 \%$ difference was reached.

(Manuscript received February 24, 2010; revision accepted for publication July 6, 2010 .) 